T. E. Cecil and P. J. Ryan

Nagoya Math. J,

Vol. 74 (1979), 67-75

\title{
DISTANCE FUNCTIONS AND UMBILIC SUBMANIFOLDS OF HYPERBOLIC SPACE
}

\author{
THOMAS E. CECIL AND PATRICK J. RYAN
}

In 1972, Nomizu and Rodriguez [5] found the following characterization of the complete umbilic submanifolds of Euclidean space.

Theorem A. Let $M^{n}, n \geq 2$, be a connected, complete Riemannian manifold isometrically immersed in a Euclidean space $E^{m}$. Every Morse function of the form $L_{p}$ has index 0 or $n$ at all of its critical points if and only if $M^{n}$ is embedded as a Euclidean n-subspace or a Euclidean nsphere in $E^{m}$.

Here $L_{p}$ is the Euclidean distance function, $L_{p}(x)=|p-f(x)|^{2}$, where $f$ is the immersion of $M^{n}$ into $E^{m}$.

Cecil [2] characterized the metric spheres in hyperbolic space $H^{m}$ in terms of hyperbolic distance functions $L_{p}$ as follows.

Theorem B. Let $M^{n}, n \geq 2$, be a connected, compact, differentiable manifold immersed in $\mathrm{H}^{m}$. Every Morse function $L_{p}$ has exactly two critical points if and only if $M^{n}$ is embedded as a metric $n$-sphere in $H^{m}$.

In $[2$, p. 351$]$, it was pointed out that the non-compact complete umbilic submanifolds in hyperbolic space could not be distinguished in terms of the distance functions alone. The purpose of this paper is to obtain the appropriate analogue of Theorem A for hyperbolic space through the introduction of two new natural classes of Morse functions.

Each of these classes is related to a type of umbilic hypersurface as follows. A function of the first new class will have as level sets a totally

Received January 11, 1978.

AMS (MOS) subject classifications (1970). Primary 53C40 and 53B25.

During the period of this research, the authors were supported by the following grants.

T. E. Cecil, NSF Grant Nos. MPS75-12825 and MCS76-07044.

P. J. Ryan, NSF Grant No. MSC76-06930, and an Indiana University Summer Faculty Fellowship. 
geodesic hyperplane and its family of equidistant hypersurfaces. A function of the second new class will have a family of horospheres as level sets. Both new classes are similar in certain ways to Euclidean linear height functions, but not in others. The new functions complement the $L_{p}$ functions whose level sets are families of hyperspheres, the third type of umbilic hypersurface in hyperbolic space.

In the course of the paper, we prove an index theorem giving the location and index of non-degenerate critical points for the new functions. This not only allows one to characterize the umbilic submanifolds, but also enables one to define tight immersions into hyperbolic space in a way similar to the Euclidean case, i.e. require that every Morse function of the first new class have the minimum number of critical points. In a subsequent article, we will discuss tight immersions into hyperbolic space in more detail (to appear in J. London Math. Soc. 19 (1979)).

\section{Complete umbilic hypersurfaces as level sets}

In this section, we introduce the two new types of distance functions, which together with the $L_{p}$, allow us to characterize spheres, horospheres and equidistant hypersurfaces as level sets. We also observe that these hypersurfaces occur in parallel families.

We first recall that the real hyperbolic space $H^{m}$ may be represented as follows (for more detail, see [4, vol. II, p. 268]). Consider $\boldsymbol{R}^{m+1}$ with a natural basis $e_{1}, \cdots, e_{m+1}$ and a nondegenerate quadratic form $b$ defined by

$$
b(x, y)=\sum_{i=1}^{m} x^{i} y^{i}-x^{m+1} y^{m+1}, \quad \text { for } x=\sum_{i=1}^{m+1} x^{i} e_{i}, y=\sum_{i=1}^{m+1} y^{i} e_{i} .
$$

Then $H^{m}$ is the hypersurface,

$$
\left\{x \in \boldsymbol{R}^{m+1} \mid b(x, x)=-1, x^{m+1}>0\right\},
$$

on which the restriction of $b$ is a positive definite metric of constant sectional curvature -1 .

For $p$ in $H^{m}$, define a function $L_{p}$ on $H^{m}$ by,

$$
L_{p}(x)=\left(\cosh ^{-1}(-b(x, p))\right)^{2} \quad \text { for } x \in H^{m},
$$

i.e. $L_{p}(x)$ is the square of the distance in $H^{m}$ from $p$ to $x$. Let

$$
S(p, r)=\left\{x \in H^{m} \mid L_{p}(x)=r^{2}\right\} .
$$


Then for $0<r<\infty$,

(1) $S(p, r)$ is a compact, umbilic hypersurface of constant curvature $-1 / \sinh ^{2} r$.

(2) The pencil of geodesics through $p$ is orthogonal to $S(p, r)$.

(3) The distance from $S(p, r)$ to $S(p, s)$ is $|r-s|$ for $0<s<\infty$.

(4) Every complete umbilic hypersurface of positive curvature is of the form $S(p, r)$ with $p$ and $r$ being uniquely determined.

We now characterize the hyperplanes (i.e. totally geodesic $H^{m-1}$ in $\left.H^{m}\right)$ and their equidistant hypersurfaces as level sets. Let

$$
\Sigma^{m}=\left\{x \in \boldsymbol{R}^{m+1} \mid b(x, x)=1\right\} .
$$

For each hyperplane $\pi$ there exists a point $\sigma$ in $\Sigma^{m}$ such that

$$
\pi=\left\{x \in H^{m} \mid b(x, \sigma)=0\right\} .
$$

The point $\sigma$ is called a pole of the hyperplane $\pi$, and $\sigma$ is determined by $\pi$ up to a sign. Fix a choice $\sigma$ of pole of $\pi$, and let

$$
L_{\pi}(x)=\sinh ^{-1}(-b(x, \sigma)), \quad \text { for } x \in H^{m},
$$

i.e. $L_{\pi}(x)$ is the distance from $x$ to the hyperplane $\pi$, and let

$$
\Omega(\sigma, r)=\left\{x \in H^{m} \mid L_{\pi}(x)=r\right\} .
$$

Thus $\pi=\Omega(\sigma, 0)$. Then for $-\infty<r<\infty$,

(1) $\Omega(\sigma, r)$ is a complete umbilic hypersurface of constant curvature $-1 / \cosh ^{2} r$.

(2) The ultraparallel pencil of geodesics determined by $\sigma$ is orthogonal to $\Omega(\sigma, r)$.

(3) The distance from $\Omega(\sigma, r)$ to $\Omega(\sigma, s)$ is $|r-s|$ for $-\infty<s<\infty$.

(4) Every complete umbilic hypersurface of negative curvature is of the form $\Omega(\sigma, r)$, with $\sigma$ and $r$ being determined up to a sign. In fact $\Omega(-\sigma,-r)=\Omega(\sigma, r)$, and a choice of either $\sigma$ or $r$ forces the choice of the other.

Finally, we consider the horospheres, i.e. complete flat umbilic hypersurfaces of $H^{m}$. Consider the cone with vertex removed,

$$
V^{m}=\left\{x \in \boldsymbol{R}^{m+1} \mid b(x, x)=0, x^{m+1}>0\right\} .
$$

One shows by an elementary argument that if $v \in V^{m}, x \in H^{m}$, then $b(x, v)<0$. Let $h$ be an arbitrary horosphere in $H^{m}$. Then there is an 
unique $v \in V^{m}$ such that

$$
h=\left\{x \in H^{m} \mid b(x, v)=-1\right\} .
$$

Let

$$
L_{h}(x)=\log (-b(x, v)) \quad \text { for } x \in H^{m},
$$

i.e. $L_{h}(x)$ is the distance from $x$ to the horosphere $h$, and let

$$
H(v, r)=\left\{x \in H^{m} \mid L_{h}(x)=r\right\} \text {. }
$$

Thus $h=H(v, 0)$. Then for $-\infty<r<\infty$,

(1) $H(v, r)$ is a horosphere.

(2) The parallel pencil of geodesics determined by $v$ is orthogonal to $H(v, r)$.

(3) The distance from $H(v, r)$ to $H(v, s)$ is $|r-s|$ for $-\infty<s<\infty$.

(4) Every horosphere occurs as an $H(v, r)$. However, $v$ is determined only up to a positive scalar factor. Once $v$ is chosen, $r$ is uniquely determined.

Remark 1. Readers familiar with the relationship between classical projective and hyperbolic geometry will have no difficulty with the notion of pencil. For the benefit of those without such experience, we provide more detail here. Let $q$ be an arbitrary point of $\boldsymbol{R}^{m+1}-\{0\}$. For each $x \in H^{m}$ which is not a multiple of $q$, we get a unique geodesic consisting of all linear combinations of $x$ and $q$ which happen to lie in $H^{m}$. The set of all these geodesics is called the pencil determined by $q$. Since all multiples of $q$ determine the same pencil, we may assume that $q$ lies in $H^{m}, \Sigma^{m}$ or $V^{m}$. As far as the geometry of $H^{m}$ is concerned, these three types of $q$ yield three types of pencils. Specifically,

(1) If $q \in H^{m}$, the pencil consists of all geodesics through $q$.

(2) If $q \in \Sigma^{m}$, the pencil consists of all geodesics orthogonal to the hyperplane $\Omega(q, 0)$, and is called a pencil of ultraparallels.

(3) If $q \in V^{m}$, no two geodesics of the pencil intersect or have a common orthogonal hyperplane, and the pencil is called a pencil of parallels.

\section{Complete umbilic hypersurfaces as orbits}

For each pencil $P$ of geodesics in $H^{m}$, let $G(P)$ be the group of isometries generated by the reflections 


$$
x \rightarrow x-2 b(x, \xi) \xi,
$$

where $b(\xi, \xi)=1$, and $b(\xi, q)=0$, where $q$ is the point of $\boldsymbol{R}^{m+1}$ determining the pencil. These are just the reflections in hyperplanes containing lines of the pencil. The following can be easily checked.

Proposition 2. (1) If $P$ is a pencil of geodesics through $p \in H^{m}$, then the orbits of $G(P)$ are $\{p\}$ and the $S(p, r)$.

(2) If $P$ is the ultraparallel pencil determined by $\sigma \in \Sigma^{m}$, then the orbits of $G(P)$ are precisely the $\Omega(\sigma, r)$.

(3) If $P$ is the parallel pencil determined by $v \in V^{m}$, then the orbits of $G(P)$ are precisely the horospheres $H(v, r)$.

Remark 3. If $P$ is a pencil of parallels or a pencil of geodesics through a point $q \in H^{m}$, then $G(P)$ coincides with the group $\hat{G}(P)$ of all isometries which leave the pencil $P$ invariant. If $P$ is a pencil of ultraparallels determined by $\sigma \in \Sigma^{m}$, then $G(P)$ is a subgroup of index 2 in $\hat{G}(P)$, the other coset being represented by the reflection

$$
x \rightarrow x-2 b(x, \sigma) \sigma
$$

in the hyperplane orthogonal to $P$.

\section{The index theorem for distance functions}

Because of the local nature of the following theorem, we shall assume that $M$ is an embedded submanifold of $H^{m}$. Let $L$ be a distance function of one of the three types defined in section 1 .

Theorem C. (Index theorem for distance functions). Let $x \in M$.

(i ). $x$ is a critical point of $L$ if and only if there is a unit normal $\xi$ to $M$ at $x$ such that

$$
\begin{aligned}
& \text { (a) } p=\cosh t x+\sinh t \xi \text {, for some } t \geq 0 \text {, if } L=L_{p}, \\
& \text { (b) } \sigma=\sinh t x+\cosh t \xi \text {, for some } t \in R \text {, if } L=L_{\pi} \text {, where } \pi \\
& =\Omega(\sigma, 0) \text {. } \\
& \text { (c) } v=t(x+\xi), \text { for some } t>0 \text {, if } L=L_{h} \text {, where } h=H(v, 0) .
\end{aligned}
$$

(ii). With $\xi$ and $t$ as in (i), $x$ is a degenerate critical point of $L$ if and only if there is an eigenvalue $\lambda$ of $A_{\xi}$ such that,
(a) $\lambda=\operatorname{coth} t \quad$ if $L=L_{p}$,
(b) $\lambda=\tanh t \quad$ if $L=L_{\pi}$,
(c) $\lambda=1 \quad$ if $L=L_{h}$. 
(iii). If $x$ is a non-degenerate critical point of $L$, then the index of $L$ at $x$ is equal to the number of eigenvalues $\lambda_{i}$ of $A_{\xi}$, counting multiplicities, such that,
(a) $\lambda_{i}>\operatorname{coth} t \quad$ if $L=L_{p}$,
(b) $\lambda_{i}>\tanh t \quad$ if $L=L_{\pi}$,
(c) $\lambda_{i}>1 \quad$ if $L=L_{h}$.

The theorem was proven for the $L_{p}$ functions by Cecil [2, p. 245]. The proofs in the other cases are very similar and are thus omitted. By using standard methods involving focal points and Sard's theorem, Cecil showed that the set of $p \in H^{m}$ such that $L_{p}$ is not a Morse function has measure zero in $H^{m}$. The following corresponding result for $L_{\pi}$ and $L_{h}$ is proven by similar methods, and we give only an outline of the proof.

Proposition 4. (a) The set of $\sigma \in \Sigma^{m}$ such that $L_{\pi}$, where $\pi=\Omega(\sigma, 0)$, is not a Morse function has measure zero in $\Sigma^{m}$.

(b) The set of $v \in V^{m}$ such that $L_{h}$, where $h=H(v, 0)$, is not a Morse function has measure zero in $V^{m}$.

Proof. (a) Let $\nu(M)$ denote the bundle of unit normal vectors to $M$ in $H^{m}$. Define a map,

$$
F: \nu(M) \times R \rightarrow \Sigma^{m},
$$

by

$$
F(x, \xi, t)=\sinh t x+\cosh t \xi
$$

A straightforward calculation similar to the proof of Proposition 1 of [2, p. 343] shows that $F$ has a critical point at $(x, \xi, t)$ if and only if $\tanh t$ is an eigenvalue of $A_{\xi}$. Comparing this with the index theorem, one sees that $L_{\pi}$ has a degenerate critical point if and only if $\sigma$ is a critical value of $F$, where $\pi=\Omega(\sigma, 0)$. By Sard's theorem, the set of critical values of $F$ has measure zero in $\Sigma^{m}$, and the result follows.

(b) Define a map

$$
G: \nu(M) \times(0, \infty) \rightarrow V^{m}
$$

by

$$
G(x, \xi, t)=t(x+\xi) .
$$

As above, a direct computation shows that $(x, \xi, t)$ is a critical point of 
$G$ if and only if 1 is an eigenvalue of $A_{\xi}$. The result follows through comparison with the index theorem and use of Sard's theorem.

Using Proposition 4, one can prove the following useful proposition analogous to the lemma of Nomizu and Rodriguez [5, p. 199]. The proof, which we omit, is similar to that of [5].

Proposition 5. (a). Suppose $L_{\pi}$ has a non-degenerate critical point of index $k$ at $x \in M$, and suppose $\pi=\Omega(\sigma, 0)$ for $\sigma \in \Sigma^{m}$. Then there is a point $\sigma^{\prime} \in \Sigma^{m}$ such that $L_{\pi^{\prime}}$, with $\pi^{\prime}=\Omega\left(\sigma^{\prime}, 0\right)$, is a Morse function having a critical point $y \in M$ of index $k$ ( $\sigma^{\prime}$, and $y$ may be chosen as close to $\sigma$ and $x$, respectively, as desired).

(b) Suppose $L_{h}$ has a non-degenerate critical point of index $k$ at $x \in M$, and suppose $h=H\left(v^{\prime}, 0\right)$ for $v \in V^{m}$. Then there is a point $v^{\prime} \in V^{m}$ such that $L_{h^{\prime}}$, with $h^{\prime}=H\left(v^{\prime}, 0\right)$, is a Morse function having a critical point $y \in M$ of index $k$ ( $v^{\prime}$ and $y$ may be chosen as close to $v$ and $x$, respectively, as desired).

\section{The characterization in terms of Morse theory}

We now prove the main characterization theorem.

Theorem D. Let $M^{n}, n \geq 2$, be a connected, complete Riemannian manifold isometrically immersed in $H^{m}$. Every Morse function of the form $L_{p}$ or $L_{\pi}$ has index 0 or $n$ at all its critical points if and only if $M^{n}$ is embedded as a sphere, horosphere, or equidistant hypersurface in a totally geodesic $H^{n+1} \subset H^{m}$.

Proof. If $M^{n}$ is one of the submanifolds listed, then $M^{n}$ is umbilic. It follows from Theorem $\mathrm{C}$ that all of the indices in question must be 0 or $n$.

Conversely, under the assumption on the indices of the critical points of the $L_{p}$ and $L_{\pi}$, we will prove that $M^{n}$ must be umbilic. A modification of Cartan's proof in the Euclidean case [1, p. 231] then shows that a complete umbilic submanifold is embedded as one of the three standard models in a totally geodesic $H^{n+1}$.

As before, because of the local nature of the calculation, we treat $M^{n}$ as an embedded submanifold and dispense with mentioning the immersion.

Let $x$ be an arbitrary point of $M^{n}$, and $\xi$ a unit normal to $M^{n}$ at $x$. Let $\lambda$ be the eigenvalue of $A_{\xi}$ with largest absolute value. If $\lambda=0$, then 
all of the eigenvalues are equal as desired. If not, we may assume that $\lambda>0$, otherwise, we simply change the sign of $\xi$.

If $\lambda>1$, the proof of Proposition 4 of [2] using the index theorem for the $L_{p}$ functions shows that $A_{\xi}=\lambda I$. If $0<\lambda \leq 1$, we use the $L_{\pi}$ functions in a similar way to get the result. To be specific, let $\mu$ be the second largest eigenvalue if such exists. Choose $t$ such that

$$
\mu<\tanh t<\lambda \text {. }
$$

Let $\sigma \in \Sigma^{m}$ be given by

$$
\sigma=\sinh t x+\cosh t \xi,
$$

and let $\pi=\Omega(\sigma, 0)$. By (iii) (b) of Theorem C, $L_{\pi}$ has a nondegenerate critical point of index $k$ at $x$, where $k$ is the multiplicity of $\lambda$. By Proposition 5 , there is a Morse function $L_{\pi}$, having a critical point of index $k$ near $x$. By the hypotheses, we must have $k=n$, and thus $A_{\xi}=\lambda I$. Hence, $M^{n}$ is an umbilic submanifold.

Remark 6. The theorem could also be formulated using the $L_{h}$ functions as well as the others, i.e. every Morse function of the form $L_{p}, L_{\pi}$ or $L_{h}$ has index 0 or $n$ at all of its critical points if and only if $M^{n}$ is an umbilic submanifold. The fact that the $L_{h}$ are not needed in the characterization is similar to the Euclidean situation, where it is not necessary to use the linear height functions to characterize the umbilic submanifolds. Geometrically, the $L_{h}$ are not necessary because a horosphere can be approximated arbitrarily closely by a sphere, and likewise by an equidistant hypersurface.

\section{Concluding remarks}

The framework developed here can also be used to encompass the Euclidean and spherical cases. We list the results for completeness.

In the Euclidean case, $L_{p}(x)=|x-p|^{2}$ and $L_{n}(x)=\langle x, \sigma\rangle$, where $\sigma$ is any unit normal to the hyperplane $\pi$. The level sets are the spheres centered at $p$, and the planes parallel to $\pi$, respectively. There are two kinds of pencils, namely, all lines through a point, and all lines orthogonal to a hyperplane. Each complete umbilic hypersurface is an orbit of the group of isometries $G(P)$ generated by reflections in hyperplanes containing lines of $P$. In the notation of Section 2 , if $P$ is a pencil of lines orthogonal to hyperplane, then $\hat{G}(P)$ includes, in addition, the reflec- 
tions in hyperplanes orthogonal to $P$. Thus, $\hat{G}(P) / G(P) \cong \boldsymbol{R}$.

In the sphere, there is essentially one kind of distance function and one kind of pencil. A hyperplane in $S^{m}$ with pole $p \in S^{m}$ is of the form

$$
\pi=\left\{x \in S^{m} \mid\langle x, p\rangle=0\right\} .
$$

The geodesics orthogonal to $\pi$ coincide with the geodesics through $p$, and the collection of level sets of $L_{p}$ and $L_{x}$ are the same, i.e. the spheres centered at $p$. Finally, a sphere centered at $p$ is an orbit of the group $G(P)$ while $G(P)$ is of index 2 in $\hat{G}(P)$.

Noting that on $S^{m}$,

$$
\langle x, p\rangle=1-\frac{1}{2}|x-p|^{2},
$$

we see that the following Morse theoretic characterization of metric spheres in $S^{m}$ follows directly from the Euclidean Theorem A.

Theorem E. Let $M^{n}, n \geq 2$, be a connected, complete Riemannian manifold isometrically immersed in $S^{m}$. Every Morse function of the form $L_{p}$ has index 0 or $n$ at any of its critical points if and only if $M^{n}$ is embedded as a metric sphere $S^{n}$.

Finally, we note that results of this nature for complex submanifolds of complex projective space may be found in [3].

\section{REFERENCES}

[1] E. Cartan, Leçons sur la géométrie des espaces de Riemann, deuxième édition, Gauthier-Villars, Paris, 1946.

[2] T. Cecil, A characterization of metric spheres in hyperbolic space by Morse theory, Tohoku Math. J. 26 (1974), 341-351.

[ 3 ] — - Geometric applications of critical point theory to submanifolds of complex projective space, Nagoya Math. J. 55 (1974), 5-31.

[4] S. Kobayashi and K. Nomizu, Foundations of differential geometry, Vo. II, John Wiley and Sons, Inc., New York, 1969.

[5] K. Nomizu and L. Rodriguez, Umbilical submanifolds and Morse functions, Nagoya Math. J. 48 (1972), 197-201.

Department of Mathematics

College of the Holy Cross

Department of Mathematics

Indiana University at South Bend 